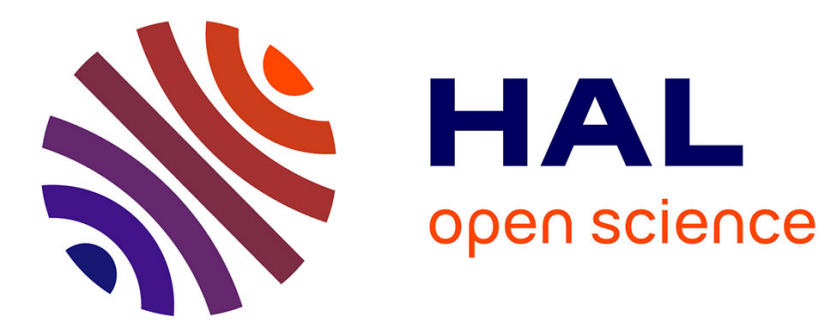

\title{
On the reflection of acoustic waves on a rough surface
}

Maurice A. Biot

\section{To cite this version:}

Maurice A. Biot. On the reflection of acoustic waves on a rough surface. Journal of the Acoustical Society of America, 1958, 30 (5), pp.479-480. hal-01368686

\section{HAL Id: hal-01368686 https://hal.science/hal-01368686}

Submitted on 21 Sep 2016

HAL is a multi-disciplinary open access archive for the deposit and dissemination of scientific research documents, whether they are published or not. The documents may come from teaching and research institutions in France or abroad, or from public or private research centers.
L'archive ouverte pluridisciplinaire HAL, est destinée au dépôt et à la diffusion de documents scientifiques de niveau recherche, publiés ou non, émanant des établissements d'enseignement et de recherche français ou étrangers, des laboratoires publics ou privés. 


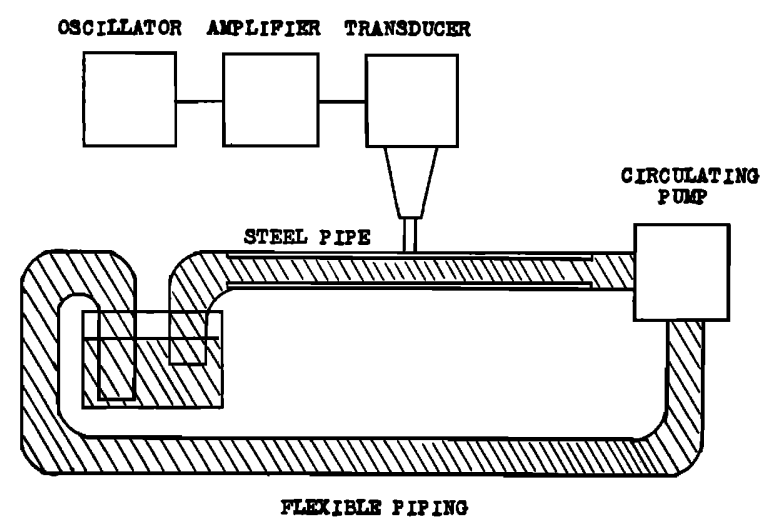

Fig. 2. Flowing system.

the fact that the dispersed particles are denser than the medium). The dropping of the coagulated water and rust particles to the bottom of the beaker can be easily seen. If a beam of light is directed through the beaker, the agglomeration of the suspended particles is also visible. A small screw (3/8-in. base) can coagulate effectively a rather large amount of liquid $(500 \mathrm{cc})$. No noticeable heat is generated in the liquid at all because the power is too low.

The effective coagulation is complete in about $30 \mathrm{sec}$, the larger particles dropping out and the smaller ones agglomerating into larger, statically charged particles, which remain in suspension because of agitation. Before irradiation, the water particles averaged $10 \mu$ and the rust particles $20 \mu$ in size (measured under a microscope). After one minute of irradiation, a great number of particles had dropped to the bottom of the beaker; the remaining water clumps averaged $200 \mu$ and rust $90 \mu$, with very little rust left at all. Whereas, before irradiation, the microscopic fields
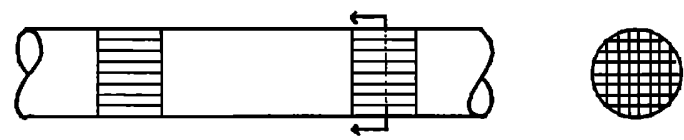

Fig. 3. Baffle system.

were completely filled wtih water and rust particles, only 2 to 4 water clumps were present in each field after irradiation. Several fields had to be examined to find rust particles. These remaining particles were practically all in the bottom layers of the fuel. Microscopic examination of the upper layers revealed a few particles present only after extensive search of many portions.

The coagulated particles which were still suspended adhered to glass or metal surfaces, preferably to rough or pitted surfaces. Therefore, by transferring the treated fuel from beaker to beaker, the particles remained behind on the surfaces and a liquid, clear to the naked eye, was obtained.

A second series of experiments was run in which the head of the screw was soldered to the outside of a steel pipe (see Fig. 2). This pipe was attached to a sigma pump by means of tygon tubing. The emulsified fuel was pumped through the pipe, with the transducer vibrating, at a rate of $800 \mathrm{cc} / \mathrm{min}$ through tubing of 1-in. inside diameter. The results were the same as in the first series of experiments. The particles had coagulated so that by transferring the liquid through a series of beakers a fuel, clear to the naked eye, was obtained. Adherence of large amounts of coagulated particles to the sides of the tubing was also noticed.

To effect the coagulation, separation, and clearing in one step the following design for a pilot model piping system is suggested (see Fig. 3). This design has not been tried out in this laboratory. The pipe should contain a system of baffles of metal or glass, preferably pitted steel. For a pipe of about 6-in. diam, the baffles should be about 5 in. deep. If the piping system is a short one, the baffles should be spaced 5 in. apart; if a long one the baffles could be 10 or 20 in. apart, depending on the specific system. It is also recommended that the transmitting metal screws or bars be welded from the transducer to the pipe.

* The opinions or assertions in this paper are those of the authors and are not to be construed as official or reflecting the views of the Naval Service at large.

$\uparrow$ Physical Chemistry Section, Naval Materiel Laboratory.

Patrical Insulation Section, Naval Materiel Laboratory.

iF. Nesh, Anal. Chem. 27, 1842, 1955.

\section{On the Reflection of Acoustic Waves on a Rough Surface}

M. A. Biot

Shell Development Company, New York, New York (Received February 24, 1958)

T $\mathrm{N}$ a previous paper ${ }^{1}$ we derived closed form expressions for the 1 reflection on a rough surface of acoustic waves generated by a simple harmonic point source. Because of an error in sign for $\sigma$ the problem which has been solved in the above paper corresponds to acoustic waves in a liquid being reflected on a perfectly rigid smooth surface covered with small air bubbles. This is easily seen if we remember that the dipole induced in the air bubble is of opposite sign to that induced in a solid protuberance. Quantitatively in this case we must also replace $\frac{1}{2} \sigma$ by $\sigma$ and the value of $k$ by $k=1-\left(\pi^{2} / 2\right)\left(a^{3} / b^{3}\right)$.

The actual problem intended in reference 1 is that of reflection on a rigid surface with rigid bosses. In this case the boundary condition (2.18) of reference 1 must undergo a change in sign and read

$$
\begin{aligned}
\frac{\partial \phi}{\partial z} & =-\frac{1}{2} \sigma\left(\frac{\partial^{2}}{\partial z^{2}}+k^{2}\right) \phi \\
\sigma & =2 \pi N a^{3} / \kappa .
\end{aligned}
$$

A plane wave when reflected on the rough surface is multiplied by $\exp (2 i \psi)$ where the phase angle $\psi$ is given by

$$
\tan \psi=-\frac{1}{2} \sigma k \frac{\sin ^{2} \theta}{\cos \theta}
$$

with $\theta$ the angle of incidence. Except for the change in sign this phase angle is the same as given by Eq. (5.17) of reference 1 .

An interesting feature resulting from the change in sign is the appearance of a surface wave. This corresponds to a solution

$$
\phi=e^{-2 \beta} e^{-i l x}
$$

where $\beta$ is real positive. It must satisfy the wave equation and the boundary condition (1). Hence

$$
\begin{aligned}
& \beta^{2}+k^{2}=l^{2} \\
& \beta=\frac{1}{2} \sigma\left(\beta^{2}+k^{2}\right)
\end{aligned}
$$

or

$$
\begin{aligned}
\beta & =\frac{1}{2} \sigma l^{2} \\
k^{2} & =l^{2}\left(1-\frac{1}{4} \sigma^{2} l^{2}\right) .
\end{aligned}
$$

For the theory to be valid the wavelength along the surface must be large compared to the size of the roughness. Referring to the definition of $\sigma$ this means $\sigma l<\frac{1}{2}$. For any given value $k^{2}<\left(1 / \sigma^{2}\right)$ the second Eq. (6) has two real roots for $l^{2}$. However the largest of the two must be dropped because it corresponds to a wavelength which is not large enough relative to the roughness for the theory to be applicable. Hence for a given frequency there is only one surface wave. The phase velocity of the surface wave is

$$
v=k c / l=c\left(1-\frac{1}{4} \sigma^{2} l^{2}\right) \text {. }
$$

Since $\sigma l<\frac{1}{2}$, there is a slight dispersion.

The reflected waves due to a point source are obtained by changing $\sigma$ to $-\sigma$ in expression (3.9) of reference 1 . The velocity potential $\phi_{r}$ of the reflected field is

$$
\phi_{\mathrm{r}}=D \int_{0}^{\infty} \frac{1}{\mu+\frac{1}{2} \sigma l^{2}} J_{0}(l r) e^{-\mu(z+h)} l d l .
$$


The change in sign introduces real roots for $l$ in the denominator. These poles correspond to the surface waves generated by the point source. The solution of the integral has to be modified accordingly.

1 M. A. Biot, J. Acoust. Soc. Am. 29, 1193-1200 (1957).

\section{Supplementary Notes to "Field of a Spatially Extended Moving Sound Source"* $\dagger$}

HANS L. OESTREICHER

Wright Air Development Center, Wright-Patterson Air Force Base, Ohio (Received February 26, 1958)

$\mathrm{F}$

OLLOWING the derivation of a generalized Helmholtz formula, Eq. (S14) $\ddagger$ and a multipole expansion, Eq. (S20), for the field of a spatially extended moving sound source, the field of a sufficiently small source was discussed, just as for the source at rest, ${ }^{1}$ under the assumption that $\partial \phi / \partial n$ is a given function on $S$ which is essentially (i.e., except for simple proportionality) independent of the frequency $f$ and of the diameter of the source $a$. This condition is, of course, reasonable for sources at rest (in this case $\partial \phi / \partial n$ equals the normal velocity of the surface of the source) and is also satisfied for many important source types in motion. However, it excludes a variety of moving source types from discussion, because of the fact that the boundary condition for the moving source is given by*

$$
\partial \phi / \partial n=-(\operatorname{grad} F)-3\left(i \omega A+\operatorname{grad} \phi_{0} \cdot \operatorname{grad} A\right) .
$$

In this equation $A$ is the negative normal displacement of $S$, which can reasonably be assumed to be essentially independent of $f$ and $a$, but the second term $\operatorname{grad} \phi_{0} \cdot \operatorname{grad} A$ will, in general, have the order of magnitude $U \cdot A / a$ (see example below). Therefore $\partial \phi / \partial n$ is, in general, the sum of two terms which differ in order of magnitude by one power of $a k$.

The physical significance of this second term is a contribution of certain torsional motions of $S$ to the sound field. If the vibrations are, for instance, strictly normal to $S$, then $\operatorname{grad} A=0$ and this contribution disappears. However, an additional wave is generated if the angle between the normal of a surface element $d S$ and the Mach vector of the basic stream changes during the vibration.

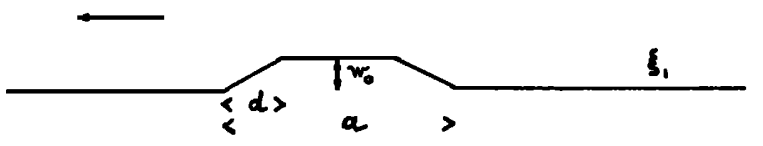

FIG. 1. Cross section along $\xi_{1}$ axis, showing velocity amplitude distribution over square membrane.

Substituting Eq. (1) in Eq. (S20) shows that the lowest term in the coefficient of the multipole of $\nu$ th order is, in general, the sum of a term of the order $(\bar{a} k)^{\nu}$ and a term of the order $(\bar{a} k)^{\nu-1}$. The first term is due to the normal velocity $-A i \omega$ of the surface $S$, while the second one appears if $\operatorname{grad} \phi_{0} \cdot \operatorname{grad} A \neq 0$. Collecting the terms of the same order in $\bar{a} k$ gives

$$
\begin{aligned}
-4 \pi \phi(x)=B_{0} g_{P}(x, \eta)+\bar{a} k\left(B_{10} g_{P}(x, \eta)+\sum\right. & \left.\left.\frac{B_{1 j}}{k} \frac{\partial g_{P}}{\partial \xi_{i}}\right|_{\xi=\eta}\right) \\
& +(\bar{a} k)^{2}(\cdots)+\cdots
\end{aligned}
$$

In this equation the coefficients $B$ are made up of integrals over $S$ and $D$, and depend of course on the shape of the source, the structure of the basic flow, and the amplitude distribution on $S$. They are all of the same order of magnitude with respect to $\vec{a} k$, under the assumptions that $A$ is essentially independent of frequency and size of the source, that $S$ is a smooth surface and that $\partial U^{2} / \partial n$ is of the order of magnitude of $U^{2} / a$. Assumptions which hold for a large class of source types. For particular shapes and types of sources one or more of the coefficients $B$, may, of course, vanish.

The first and lowest order term in (2) is a monopole. The absolute values of its normalized velocity potential $\left|4 \pi \phi / B_{0}\right|$ and pressure far field $\left|4 \pi p / \omega \rho_{0} B_{0}\right|$ are represented by the curves of Figs. S2 and S3.* This term is a pure motion effect and vanishes with decreasing speed. Although it is one order lower in $\bar{a} k$ than the next term it may often be, that this term is not predominant for acoustic frequencies because $B_{0}$ has to compete with the next term which is of the same order of magnitude as the strength of the source at rest, to which it reduces for $U=0$.

Because of this and because $B_{0}$ may be zero even for powerful sources (see example below), we have also to discuss the next term in (2) which contains a monopole and a dipole of equal order in $\bar{a} k$. In general, both of them will appear according to the coefficients $B_{10}$ and $B_{1 i}$ but for sources, like breathing spheres, where $\operatorname{grad} A=0$ it reduces to the monopole and the directivity pattern for velocity potential $\left|4 \pi \phi / a k B_{10}\right|$ and sound pressure $\left|4 \pi p / \omega \rho_{0} a k B_{10}\right|$ is again represented by Figs. S2 and S3.*

Summarizing we have, therefore, the far field of the velocity potential of a moving source which is small compared to the wavelength and which displays a monopole pattern at rest $\left(\int_{S} w d S \neq 0\right)$ is either a monopole field of the parallel stream, $g_{P}$, or the combination of a monopole and dipole field of the parallel stream. The relative intensities of monopole and dipole depend on source and speed.

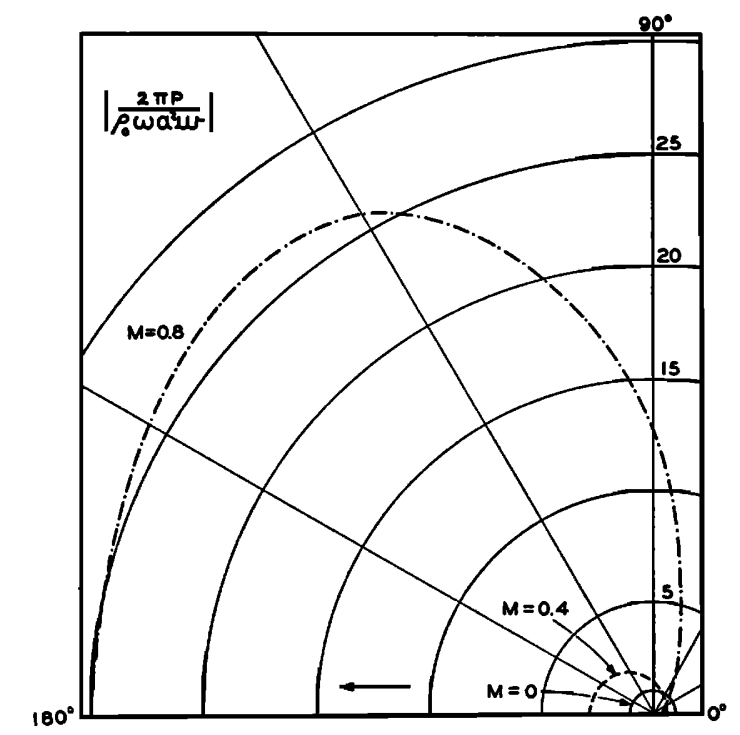

FIG. 2. Dimensionless representation of the sound pressure field of the membrane of Fig. 1 in a coordinate system rigidly attached to the source. The arrow indicates the direction of motion of the membrane.

As a typical example of a source with $B_{0}=0$, but $B_{10}$ and $B_{1 j} \neq 0$ the field of a small square membrane in an infinite baffle will now be considered. Figure 1 gives a cross section through this membrane showing the velocity amplitude $w$ as function over $S$. It is

$$
w=\left\{\begin{array}{lll}
=0 & \text { for } & \left|\xi_{1}\right| \geq \frac{a}{2} \\
=\frac{w_{0}}{d}\left( \pm \xi_{1}-\frac{a}{2}\right) & \text { for } & \frac{a}{2}-d \leq \mp \xi_{1} \leq \frac{a}{2} \\
=w_{0} & \text { for } & \left|\xi_{1}\right| \leq \frac{a}{2}-d .
\end{array}\right.
$$

The basic stream is $\phi_{0}=U x_{1}$ and $-A i \omega=w$. Due to this simplification we get $B_{0}=\int_{S}\left(\partial A / \partial \xi_{1}\right) d \xi_{1} d \xi_{2}=0$ and Eq. (2) becomes, if the 